\title{
Quantum transport in a normal metal/odd-frequency superconductor junction
}

\author{
Jacob Linder, ${ }^{1}$ Takehito Yokoyama, ${ }^{2}$ Yukio Tanaka, ${ }^{2}$ Yasuhiro Asano, ${ }^{3}$ and Asle Sudb $\emptyset^{1}$ \\ ${ }^{1}$ Department of Physics, Norwegian University of Science and Technology, N-7491 Trondheim, Norway \\ ${ }^{2}$ Department of Applied Physics, Nagoya University, Nagoya 464-8603, Japan and CREST-JST \\ ${ }^{3}$ Department of Applied Physics, Hokkaido University, Sapporo 060-8628, Japan
}

(Dated: Received October 27, 2018)

\begin{abstract}
Recent experimental results indicate the possible realization of a bulk odd-frequency superconducting state in the compounds $\mathrm{CeCu}_{2} \mathrm{Si}_{2}$, and $\mathrm{CeRhIn}$. Motivated by this, we present a study of the quantum transport properties of a normal metal/odd-frequency superconductor junctions in a search for probes to unveil the oddfrequency symmetry. From the Eliashberg equations, we perform a quasiclassical approximation to account for the transport formalism of an odd-frequency superconductor with the Keldysh formalism. Specifically, we consider the tunneling charge conductance and tunneling thermal conductance. We find qualitatively distinct behaviour in the odd-frequency case as compared to the conventional even-frequency case, in both the electrical and thermal current. This serves as a useful tool to identify the possible existence of a bulk odd-frequency superconducting state.

PACS numbers: 74.45.+c, 74.20.Rp,74.50.+r
\end{abstract}

\section{INTRODUCTION}

The symmetries of the superconducting order parameter with respect to orbital-, time-, and spin-space are governed by the Pauli principle. A wavefunction describing two electronic states must be totally antisymmetric under exchange of the particle-coordinates. This leads to a finite number of allowed combinations for the symmetries of the wavefunction. In a wide variety of superconductors ranging from those described with Bardeen-Cooper-Schrieffer/Eliashberg theory via spin-triplet superconductivity in ${ }^{3} \mathrm{He}$, to strong-coupling superconductivity in high- $T_{c}$ cuprates, the wave function of Cooper-pairs is even in the frequency domain. For such even-frequency pairing, the wavefunction may be even or odd in space depending on whether the Cooper-pairs form spinsinglets or -triplets. However, more exotic types of pairings than what is found in this wide range of materials, are in principle permitted.

Recently, it was predicted that in a ferromagnet/superconductor structure, a so-called odd-frequency pairing could take place ${ }^{1}$. Thus, the Cooper pair wavefunction is symmetric under exchange of spatial- and spin-coordinates, but antisymmetric under exchange of time-coordinates. This state had been proposed to exist by Berezinskii $\stackrel{2}{2}$ a few decades earlier in the context of liquid ${ }^{3} \mathrm{He}$, and strong experimental evidence for odd-frequency pairing now exists ${ }^{3}$. The study of such pairing in ferromagnet/conventional superconductor junctions has been addressed by a number of authors over the last years ${ }^{4}$. Furthermore, it was very recently predicted ${ }^{5,6,7}$ that due to spatial variation of the pair potential near a normal/superconductor (N/S) junction, the odd-frequency pairing state can be induced even in a conventional ballistic N/S system without spin-triplet ordering. The generation of different symmetry components and their effect on electrical transport in a normal/superconductor interface has also been studied in the diffusive limit ${ }^{8}$ in the context of the proximity effect in unconventional superconductors 9.10 .

An issue that arises in the context of the odd-frequency pairing state, is if it can be realized in a bulk superconduc- tor, i.e. without a proximity effect. There have been several theoretical proposals for this in strongly correlated systems up to now ${ }^{11,12}$. To explore an odd-frequency pairing state in heavy-fermion superconductors is an interesting topic, and an assessment of the experimental properties of $\mathrm{CeCu}_{2} \mathrm{Si}_{2}$, and $\mathrm{CeRhIn}_{5}$ concluded that odd frequency pairing may be realized in these heavy-fermion compounds ${ }^{13}$.

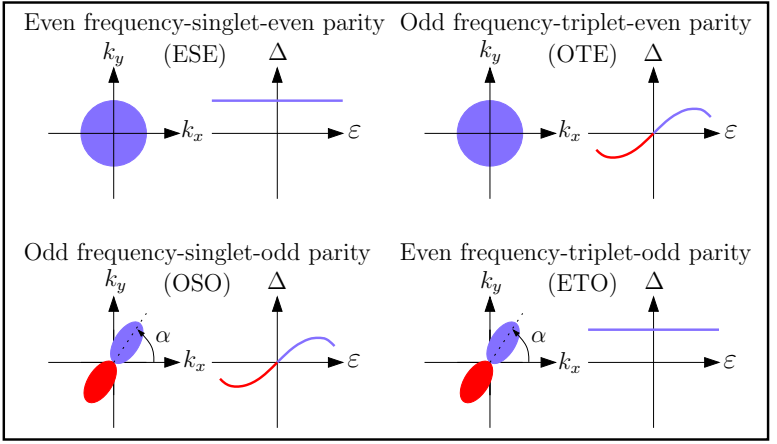

FIG. 1: (Color online) Overview of the different symmetry states we will consider in the superconducting part of the clean, twodimensional normal/superconductor junction.

However, only a very limited amount of studies have addressed the issue of identifying the odd-frequency pairing state in a bulk superconductor so far ${ }^{11,14,15}$. Hence, further clear-cut predictions are needed.

In this paper, we present the quantum transport properties of a normal metal/odd-frequency superconductor junction in the clean limit. We calculate the electrical and thermal conductances within the Blonder-Tinkham-Klapwijk (BTK) framework ${ }^{16}$ taking account of the anisotropy of the pair potential ${ }^{17}$. Our starting point is the Eliashberg equations that take into account the frequency-dependence of the pair potential. This constitutes a wide range of experimental predictions, which are routinely used to characterize superconduct-

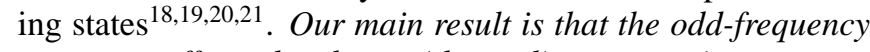
symmetry affects the charge (thermal) transport in an essen- 
tial manner at low energies (temperatures). This provides a useful tool in identifying this highly unusual superconducting state.

To elucidate the physics in a transparent manner, we employ a simple two-dimensional calculation in the clean limit. We approximate the superconducting gap with a step-function in space, which in the isotropic even-parity $s$-wave case should be an excellent approximation for low-transmission barriers. Since the low-transmission case probably is the most realistic scenario experimentally, we restrict our attention to this. In the anisotropic even-parity and odd-parity cases (corresponding e.g. to the high- $\mathrm{T}_{c}$ superconductors and $\mathrm{Sr}_{2} \mathrm{RuO}_{4}$ ), the gap may undergo a severe depletion near the barrier even for low-transmission interfaces due to the formation of zero-energy states 22 . The method used in this paper may still be able to capture qualitative features of the transport properties even in those cases, just as in the case of the $d$-wave superconductors 17 . Our results are in fact consistent with recent findings ${ }^{5}$ including a self-consistent solution of the spatial variation of the superconducting gap near the interface.

We will use boldface notation for 3 -vectors, $. \hat{\text { for }} 4 \times 4$ matrices, and ... for $2 \times 2$ matrices. Pauli-matrices in particlehole $\times$ spin (Nambu) space are denoted as $\hat{\rho}_{i}$, while Paulimatrices in spin-space are written as $\underline{\tau}_{i}$.

\section{THEORETICAL FORMULATION}

\section{A. Equations for odd-frequency superconductivity}

The frequency-dependence of the superconducting order parameter may be naturally taken into account in the approach developed by Eliashberg ${ }^{23}$, where details of the electronboson interaction are taken seriously. This contrasts the usual weak-coupling picture where the pairing interaction is taken to be constant. For our purposes, the following Hamiltonian is an appropriate starting point:

$$
\begin{aligned}
& \hat{H}=\sum_{\alpha} \int \mathrm{d} \mathbf{r} \psi_{\alpha}(\mathbf{r})^{\dagger} H_{f}(\mathbf{r}) \psi_{\alpha}(\mathbf{r})+\int \mathrm{d} \mathbf{r} b^{\dagger}(\mathbf{r}) H_{b}(\mathbf{r}) b(\mathbf{r}) \\
& +\sum_{\alpha} \iint \mathrm{d} \mathbf{r} \mathbf{d} \mathbf{r}^{\prime} \mathcal{V}\left(\mathbf{r}-\mathbf{r}^{\prime}\right) \psi_{\alpha}^{\dagger}(\mathbf{r}) \psi_{\alpha}(\mathbf{r})\left[b(\mathbf{r})+b^{\dagger}(\mathbf{r})\right],
\end{aligned}
$$

where $H_{f}$ is the Hamiltonian for free fermions which we assume may be written as $H_{f}(\mathbf{r})=-\frac{1}{2 m}(\nabla-1 e \mathbf{A})^{2}-\mu$, while $H_{b}$ is the Hamiltonian for free bosons. Above, $\alpha$ denotes the spin index while $\psi$ and $b$ are fermion and boson operators, respectively. Introducing the Fourier-transformation $b(\mathbf{r})=\frac{1}{N} \sum_{\mathbf{q}} b_{\mathbf{q}} \mathrm{e}^{-1 \mathbf{q} \cdot \mathbf{r}}, B_{\mathbf{q}}=b_{\mathbf{q}}+b_{-\mathbf{q}}^{\dagger}$, we obtain the Heisenberg equations of motion

$$
\begin{aligned}
& 1 \partial_{t} \psi_{\alpha}(\mathbf{r}, t)=H_{f}(\mathbf{r}) \psi_{\alpha}(\mathbf{r}, t)+\sum_{\mathbf{q}} \zeta(\mathbf{r}, t, \mathbf{q}) \psi_{\alpha}(\mathbf{r}), \\
& 1 \partial_{t} \psi_{\alpha}^{\dagger}(\mathbf{r}, t)=-H_{f}^{*}(\mathbf{r}) \psi_{\alpha}^{\dagger}(\mathbf{r}, t)-\sum_{\mathbf{q}} \zeta(\mathbf{r}, t, \mathbf{q}) \psi_{\alpha}^{\dagger}(\mathbf{r}),
\end{aligned}
$$

where $\zeta(\mathbf{r}, t, \mathbf{q}) \equiv \mathcal{V}_{\mathbf{q}} B_{\mathbf{q}}(t) \mathrm{e}^{-1 \mathbf{q} \cdot \mathbf{r}}$ and $\mathcal{V}_{\mathbf{q}}$ is the Fouriertransform of $\mathcal{V}\left(\mathbf{r}-\mathbf{r}^{\prime}\right)$. Note that $\mathcal{V}$ is not the effective pairing potential between electrons. Having obtained the timederivatives of the fermion operators, we may now calculate the equation of motion for the Green's functions. This procedure is standard and covered in e.g. Refs. 24, 25, 26, 27. Taking into account the effect of the electron-boson interactions explicitly in the Hamiltonian naturally includes a frequency-dependence in the effective electron-electron interaction ${ }^{23}$ which is obtained by integrating out the bosonic degrees of freedom in the partition function. The effective electron-electron interaction mediated by a boson excitation may in general be written as

$$
V(\mathbf{q}, \Omega)=\frac{2\left|\mathcal{V}_{\mathbf{q}}\right|^{2} \omega_{\mathbf{q}}}{\omega_{\mathbf{q}}^{2}+\Omega^{2}}
$$

where $\mathbf{q}=\mathbf{k}-\mathbf{k}^{\prime}$ and $\Omega=\omega-\omega^{\prime}$ are the momentum and energy transfers, respectively, of the interaction process. Above, $\omega_{\mathbf{q}}$ is the frequency of the boson propagator. Note that the pairing potential in Eq. (3) is even in $\Omega$, i.e. $V(\mathbf{q}, \Omega)=V(\mathbf{q},-\Omega)$. The self-consistency equation for the order parameter quite generally has the structure ${ }^{11}$

$$
\Delta(\mathbf{k}, \omega) \sim \sum_{\mathbf{k}^{\prime} \omega^{\prime}} \frac{V\left(\mathbf{k}-\mathbf{k}^{\prime}, \omega-\omega^{\prime}\right) \Delta\left(\mathbf{k}^{\prime}, \omega^{\prime}\right)}{\varepsilon_{\mathbf{k}}^{\prime 2}+\omega^{\prime 2}},
$$

which may be re-written as

$$
\Delta(\mathbf{k},-\omega) \sim \sum_{\mathbf{k}^{\prime} \omega^{\prime}} \frac{V\left(\mathbf{k}-\mathbf{k}^{\prime}, \omega-\omega^{\prime}\right) \Delta\left(\mathbf{k}^{\prime},-\omega^{\prime}\right)}{\varepsilon_{\mathbf{k}}^{\prime 2}+\omega^{\prime 2}},
$$

by exploiting $V(\mathbf{q}, \Omega)=V(\mathbf{q},-\Omega)$. The above equations show that both $\Delta(\mathbf{k}, \omega)=\Delta(\mathbf{k},-\omega)$ and $\Delta(\mathbf{k}, \omega)=$ $-\Delta(\mathbf{k},-\omega)$ are possible solutions of the gap equation. Therefore, although the pairing interaction is even in frequency, the gap $\Delta$ in principle may be both even or odd in frequency. In fact, it is in general a superposition of even- and oddfrequency components $\frac{11,28}{}$. Assuming that the energy transfer is small compared to the term containing the momenta in Eq. (3), $|\Omega| \ll\left|\omega_{\mathbf{q}}\right|$, one obtains a part of the pairing potential which is linear in $\omega$ and $\omega^{\prime}$ and one that is quadratic in the same quantities 11 . The former part is the necessary ingredient to obtain a superconducting order parameter that is odd-in-frequency. It is also possible to adopt a purely phenomenological approach to an odd-frequency superconductor by assuming the frequency-dependence of the gap a priori ${ }^{29}$.

Let us now consider the structure of the Green's function matrix for an odd-frequency superconductor. It is instructive to briefly mention the result for an ordinary BCS superconductor, which has an even frequency-singlet-even parity (ESE) symmetry. In the BCS case, one obtains

$$
\left(1 \frac{\partial}{\partial t_{1}} \hat{\rho}_{3}-\hat{\xi}-\hat{\Delta}\left(\mathbf{r}_{1}\right)\right) \hat{G}^{\mathrm{R}}(1,2)=\delta(1-2) \check{1} .
$$

Assuming a homogeneous and isotropic system where the Green's function only depends on the relative coordinates $t=t_{1}-t_{2}$ and $\mathbf{r}=\mathbf{r}_{1}-\mathbf{r}_{2}$, and where $\hat{\Delta}\left(\mathbf{r}_{1}\right)=\hat{\Delta}$ is 
a constant, one may Fourier-transform Eq. (6) according to $\hat{G}^{\mathrm{R}}(\mathbf{p}, \varepsilon)=\iint \mathrm{d} \mathbf{r e}^{-1 \mathbf{p r}} \mathrm{d} t \mathrm{e}^{1 \varepsilon t} \hat{G}^{\mathrm{R}}(\mathbf{r}, t)$, where $\varepsilon$ and $\mathbf{p}$ is the quasiparticle energy measured from Fermi level and momentum, respectively. We then obtain

$$
\left(\varepsilon \hat{\rho}_{3}-\hat{\xi}_{\mathbf{p}}-\hat{\Delta}\right) \hat{G}^{\mathrm{R}}(\mathbf{p}, \varepsilon)=\hat{1},
$$

which upon matrix inversion yields the well-known BCS solution. The quasiclassical Green's functions $\check{g}\left(\mathbf{p}_{\mathrm{F}}, \mathbf{r} ; \varepsilon, t\right)$ is obtained from the Gor'kov Green's functions $G(\mathbf{p}, \mathbf{r} ; \varepsilon, t)$ by integrating out the dependence on kinetic energy, assuming that $\breve{G}$ is strongly peaked at Fermi level,

$$
\check{g}\left(\mathbf{p}_{\mathrm{F}}, \mathbf{r} ; \varepsilon, t\right)=\frac{1}{\pi} \int \mathrm{d} \xi_{\mathbf{p}} \check{G}(\mathbf{p}, \mathbf{r} ; \varepsilon, t) .
$$

The above assumption is typically applicable to superconducting systems where the characteristic length scale of the perturbations present, namely superconducting coherence length, is much larger than the Fermi wavelength. The corresponding characteristic energies of such phenomena must be much smaller than the Fermi energy $\varepsilon_{\mathrm{F}}$. The quasiclassical Green's functions may be divided into an advanced (A), retarded (R), and Keldysh (K) component, each of which has a $4 \times 4$ matrix structure in the combined particle-hole and spin space. One has that

$$
\check{g}=\left(\begin{array}{cc}
\hat{g}^{\mathrm{R}} & \hat{g}^{\mathrm{K}} \\
0 & \hat{g}^{\mathrm{A}}
\end{array}\right),
$$

where the elements of $\check{g}\left(\mathbf{p}_{\mathrm{F}}, \mathbf{r} ; \varepsilon, t\right)$ read

$$
\hat{g}^{\mathrm{R}, \mathrm{A}}=\left(\begin{array}{cc}
\underline{g}^{\mathrm{R}, \mathrm{A}} & \underline{f}^{\mathrm{R}, \mathrm{A}} \\
-\tilde{\tilde{f}}^{\mathrm{R}, \mathrm{A}} & -\underline{\tilde{g}}^{\mathrm{R}, \mathrm{A}}
\end{array}\right), \hat{g}^{\mathrm{K}}=\left(\begin{array}{cc}
\underline{g}^{\mathrm{K}} & \frac{f^{\mathrm{K}}}{\underline{\tilde{f}}^{\mathrm{K}}} \\
\underline{\tilde{g}}^{\mathrm{K}}
\end{array}\right) .
$$

The quantities $\underline{g}$ and $\underline{f}$ are $2 \times 2$ spin matrices, with the structure

$$
\underline{g}=\left(\begin{array}{cc}
g_{\uparrow \uparrow} & g_{\uparrow \downarrow} \\
g_{\downarrow \uparrow} & g_{\downarrow \downarrow}
\end{array}\right) .
$$

Due to internal symmetry relations between these Green's functions, all of these quantities are not independent. In particular, the tilde-operation is defined as

$$
\tilde{f}\left(\mathbf{p}_{\mathrm{F}}, \mathbf{r} ; \varepsilon, t\right)=f\left(-\mathbf{p}_{\mathrm{F}}, \mathbf{r} ;-\varepsilon, t\right)^{*} .
$$

For a bulk $s$-wave superconductor, the retarded part may be expressed in terms of the normal and anomalous Green's functions $g$ and $f$ as follows:

$$
\hat{g}^{\mathrm{R}}=\left(\begin{array}{cc}
g \underline{1} & f_{1 \tau_{2}} \mathrm{e}^{1 \chi} \chi \\
f_{\underline{1} \tau_{2}} \mathrm{e}^{-1 \chi} & \underline{-g} \underline{1}
\end{array}\right),
$$

Here, $\chi$ is the globally broken $\mathrm{U}(1)$ phase associated with the spontaneous symmetry breaking of the superconducting state. In the odd-frequency case, however, one finally arrives at

$$
\left[\varepsilon \hat{\rho}_{3}-\hat{\xi}_{\mathbf{p}}-\hat{\Delta}(\varepsilon)\right] \hat{G}^{\mathrm{R}}(\mathbf{p}, \varepsilon)=\hat{1},
$$

where now $\hat{\Delta}(\varepsilon)$ is the odd-frequency gap matrix. Note that Eq. (14) is equivalent to the well-known Eliashberg equation. The structure of the Green's function for an odd-frequency superconductor may be different from Eq. (13) depending on the spin-symmetry. For instance, the bulk Green's function matrix for an odd-frequency spin-triplet even-parity superconductor has the structure:

$$
\hat{g}^{\mathrm{R}}=\left(\begin{array}{cc}
g \underline{1} & f \tau_{\tau_{1}} \mathrm{e}^{\mathrm{l} \chi} \\
-f \underline{\tau_{1}} \mathrm{e}^{-1 \chi} & -g \underline{1}
\end{array}\right)
$$

Performing a quasiclassical approximation on Eq. (14) yields the Eilenberger equation, which reduces to the Usadel equation in the dirty limit. Note that for both even- and oddfrequency superconducting order parameters, the pairing interaction itself is always even in the frequency coordinate.

A quite general formalism for treating quantum transport in non-uniform superconducting systems, e.g. nor$\mathrm{mal} / \mathrm{superconductor} \mathrm{heterostructures,} \mathrm{has} \mathrm{been} \mathrm{developed} \mathrm{by}$ Tanaka and co-workers ${ }^{30}$. For instance, the conductance spectra of a normal/superconductor junction may be obtained along the lines of Refs. 30,31 by numerically solving the Usadel equation using Nazarov's generalized boundary conditions ${ }^{10}$. Interestingly, taking the limit $R_{d} \rightarrow 0$ and $\theta \rightarrow 0$ in this formalism, where $R_{d}$ represents the resistance of the normal metal region and $\theta$ is a measure of the proximity effect, leads to the well-known expression for the conductance obtained in the BTK-formalism ${ }^{16}$. This may be seen specifically for the electrical conductance by consulting Eqs. (15) and (16) in Ref. 30, and for the thermal conductance in Eq. (19) of Ref. 31 Therefore, since the above treatment of the Eliashberg equation shows that the odd-frequency dependence of the gap may be taken into account simply by substituting $\Delta \rightarrow \Delta(\varepsilon)$, quantum transport for an odd-frequency superconductor can be treated in the BTK-formalism by performing the same substitution. However, the derivation of the Bogolioubov-de Gennes equation for odd-frequency superconductivity is challenging since it is not obvious how to take into account the strong retardation effects of the pairing potential.

\section{B. Transport formalism}

We adopt the Keldysh formalism using Nazarov's generalized boundary conditions 10 to obtain the electrical and thermal conductance for odd-frequency superconductors. We assume, without loss of generality, that the gap $\Delta(\varepsilon, \vartheta)$ has an opposite-spin pairing symmetry in both the singlet and triplet case. To encompass accessible experimental techniques, we will focus on two experimentally accessible quantities that encode how the odd-frequency pairing symmetry is manifested in transport properties: namely, the normalized chargeconductance $G(\mathrm{eV})$ for $T=0$ and the thermal-conductance $\kappa(T)$. The procedure for obtaining these quantities is treated in detail in Refs. 30, 31, 32. In the limit of zero resistance in 
the normal part and vanishing proximity effect, one finds

$$
\begin{aligned}
G & =\frac{1}{G_{\mathrm{N}}} \int_{-\pi / 2}^{\pi / 2} \mathrm{~d} \vartheta \cos \vartheta \Gamma_{+}(e V, \vartheta), \\
\kappa & =\int_{-\pi / 2}^{\pi / 2} \int_{-\infty}^{\infty} \mathrm{d} \vartheta \mathrm{d} \varepsilon \frac{\varepsilon^{2} \beta^{2} \Gamma_{-}(e V, \vartheta)}{4 \Delta_{0} \cosh ^{2}(\beta \varepsilon)(\cos \vartheta)^{-1}}
\end{aligned}
$$

where $G_{\mathrm{N}}$ is the normal-state conductance and we have defined

$$
\begin{gathered}
\Gamma_{\alpha}(\varepsilon, \vartheta)=1+\alpha\left|\frac{4 \Omega_{-} \tilde{\Omega}_{+} \mathrm{e}^{-1 \gamma_{+}}}{\Omega_{+} \Omega_{-}\left(4-Z_{\vartheta}^{2}\right)+Z_{\vartheta}^{2} \tilde{\Omega}_{+} \tilde{\Omega}_{-} \mathrm{e}^{1\left(\gamma_{-}-\gamma_{+}\right)}}\right|^{2} \\
-\left|\frac{2\left[\Omega_{+} \Omega_{-}\left(2+Z_{\vartheta}\right)-Z_{\vartheta} \tilde{\Omega}_{+} \tilde{\Omega}_{-} \mathrm{e}^{1\left(\gamma_{-}-\gamma_{+}\right)}\right]}{\Omega_{+} \Omega_{-}\left(4-Z_{\vartheta}^{2}\right)+Z_{\vartheta}^{2} \tilde{\Omega}_{+} \tilde{\Omega}_{-} \mathrm{e}^{1\left(\gamma_{-} \gamma_{+}\right)}}-1\right|^{2}
\end{gathered}
$$

Above, we have introduced $\vartheta_{+}=\vartheta, \vartheta_{-}=\pi-\vartheta$, and $\Omega_{ \pm}=\sqrt{\left(1+\operatorname{sign}(\varepsilon) / g_{ \pm}\right) / 2}$, where $\operatorname{sign}(\varepsilon) \rightarrow-\operatorname{sign}(\varepsilon)$ for $\Omega \rightarrow \tilde{\Omega}$. The phase of the superconducting gap is contained in the factor $\mathrm{e}^{1 \gamma_{ \pm}}=\mathrm{e}^{\mathrm{l} \gamma\left(\vartheta_{ \pm}\right)}=f_{ \pm} /\left|f_{ \pm}\right|$. The quantities $g_{ \pm}$and $f_{ \pm}$are the asymptotic values of the normal and anomalous Green's functions of the odd-frequency superconductor in a gauge where the superconducting order parameter is real: $g_{ \pm}=\varepsilon / \sqrt{\varepsilon^{2}-\left|\Delta\left(\varepsilon, \vartheta_{ \pm}\right)\right|^{2}}, f_{ \pm}=$ $\Delta\left(\varepsilon, \vartheta_{ \pm}\right) / \sqrt{\left|\Delta\left(\varepsilon, \vartheta_{ \pm}\right)\right|^{2}-\varepsilon^{2}}$. We have introduced $Z_{\vartheta}=$ $-1 Z / \cos \vartheta$, where $Z$ denotes the strength of the scattering potential near the barrier. In what follows, we fix $Z=3$, corresponding to a typical low-transparency barrier which is experimentally realistic. Note that in the expression for $\kappa$, we have considered the linear response regime for a small temperaturegradient in the system and introduced $\beta=1 / T$ where $T$ is the temperature of the reservoirs.

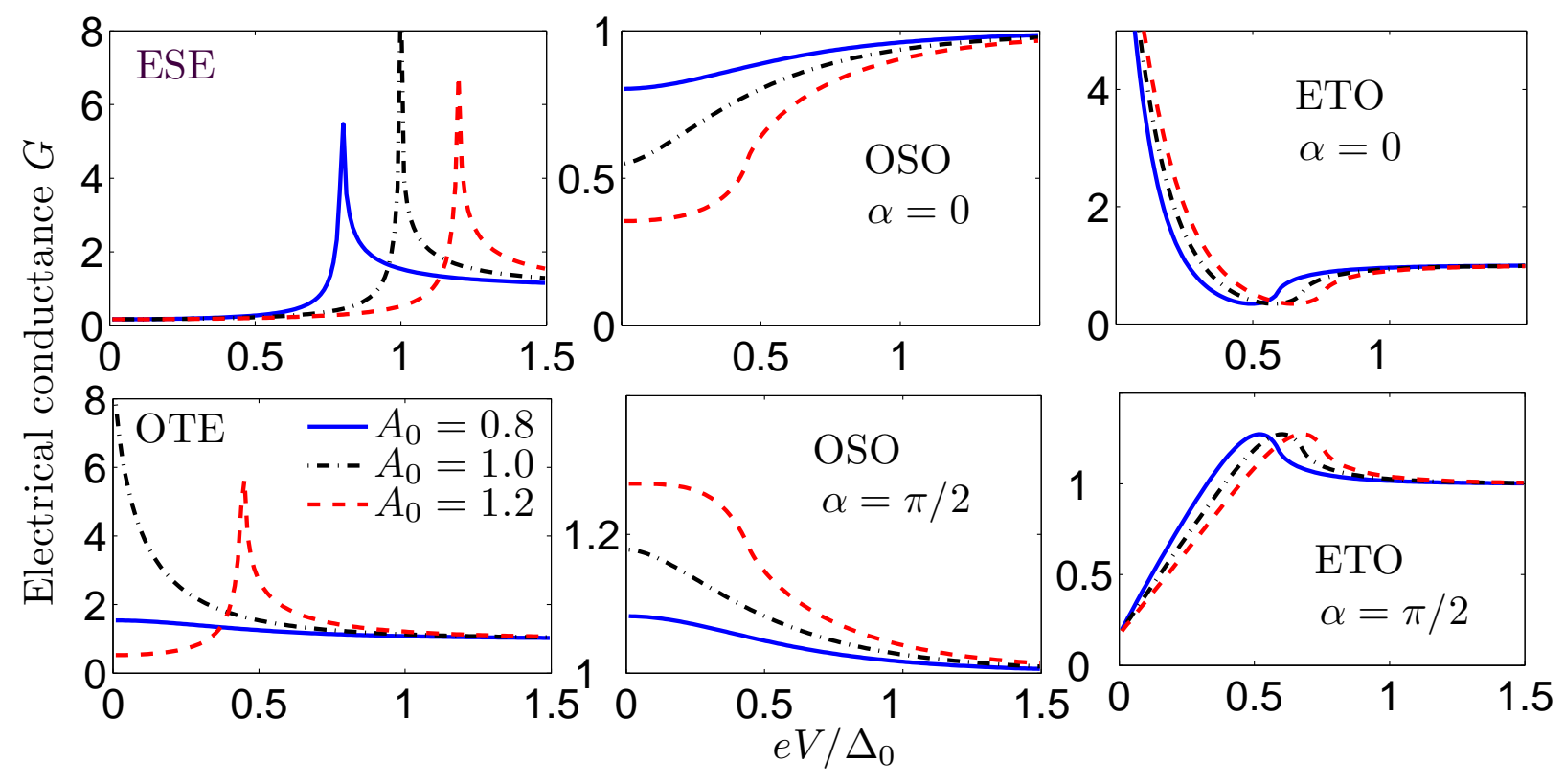

FIG. 2: (Color online) Plot of $G$ for isotropic, even parity superconductors and odd parity superconductors for both even and odd frequency pairing.

\section{RESULTS AND DISCUSSION}

Depending on the symmetries with respect to sign inversion of frequency and momentum, corresponding to $\varepsilon \rightarrow(-\varepsilon)$ and $\vartheta \rightarrow \pi+\vartheta$, the gap may be classified as seen in Tab. 【 In each case, we will model the gap $\Delta(\varepsilon, \vartheta, T)$ as illustrated in the table. In the angular dependence of the odd-parity gaps, $\alpha$ denotes the misorientation angle between the antinodes and the interface normal (see Fig. 11). The motivation for modelling the frequency-dependence of the superconducting gap as we have done in Tab. \is that it features the low-energy behaviour of the proximity-induced odd-frequency gap in dirty ferromagnet/superconductor structures ${ }^{33}$ and that it exhibits a similar energy dependence to the gap seen in strongly correlated electron systems considered in Ref. 13 .

Recently, it was demonstrated that the odd-frequency pairing is quite generally induced near the normal/superconductor interface by a fully self-consistent calculation of the superconducting correlations ${ }^{5}$. In an ETO superconductor with $\alpha=0$, corresponding to perfect formation of zero-energy states, an 
TABLE I: Overview of the specific gap forms we will consider in this paper. We model the temperature-dependence of $A(T)$ with $A(T)=$ $A_{0} \tanh \left(1.74 \sqrt{T_{c} / T-1}\right)$, and $T_{c}=\Delta_{0} / 1.76$.

\begin{tabular}{cc}
\hline \hline Symmetry & Specific gap form $\Delta(\varepsilon, \vartheta, T)$ \\
\hline ESE & $A(T) \Delta_{0}$ \\
OTE & $A(T) \varepsilon /\left[1+\left(\varepsilon / \Delta_{0}\right)^{2}\right]$ \\
OSO & $A(T) \varepsilon \cos (\vartheta-\alpha) /\left[1+\left(\varepsilon / \Delta_{0}\right)^{2}\right]$ \\
ETO & $A(T) \Delta_{0} \cos (\vartheta-\alpha) /\left[1+\left(\varepsilon / \Delta_{0}\right)^{2}\right]$ \\
\hline \hline
\end{tabular}

OTE pairing is induced near the surface. Thus, the formation of zero-energy states may be re-interpreted as a manifestation of the odd-frequency superconductivity near the inter- face. The odd-frequency symmetry may permit the existence of gapless single-particle excitations at Fermi level. On the other hand, when the nodal direction is parallel to the interface normal ( $\alpha=\pi / 2)$, only the even-frequency states exist at the interface.

In a similar manner, the OSO pairing state can be induced near the interface of a clean normal/superconductor junction when the superconductor has an ESE symmetry. One may also apply this discussion to bulk odd-frequency superconducting states. In this scenario, the ETO (ESE) pairing can be induced at the interface for an OTE (OSO) bulk superconductor ${ }^{5}$. This should have clearly observable consequences for the quantum transport properties of a normal/oddfrequency superconductor junction. We now proceed to investigate this in further detail.
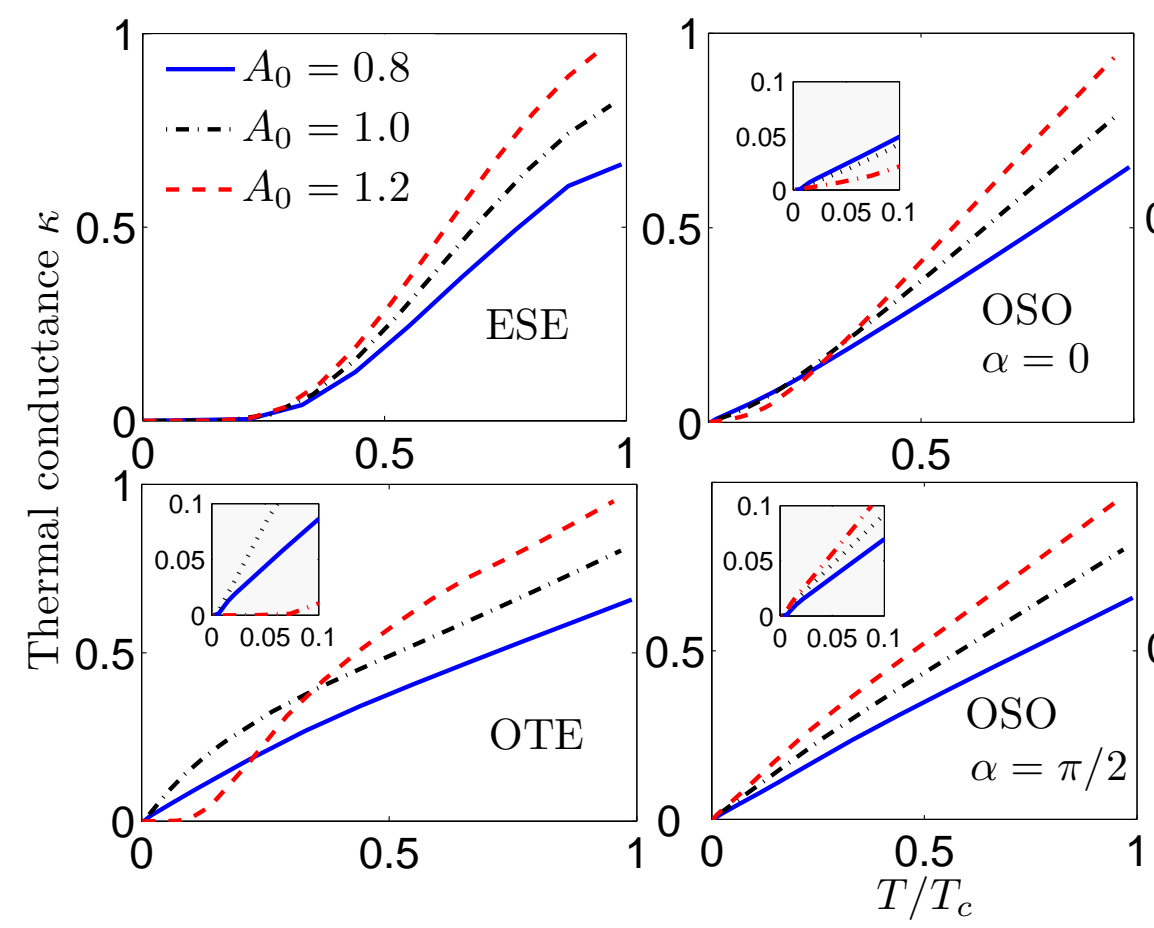
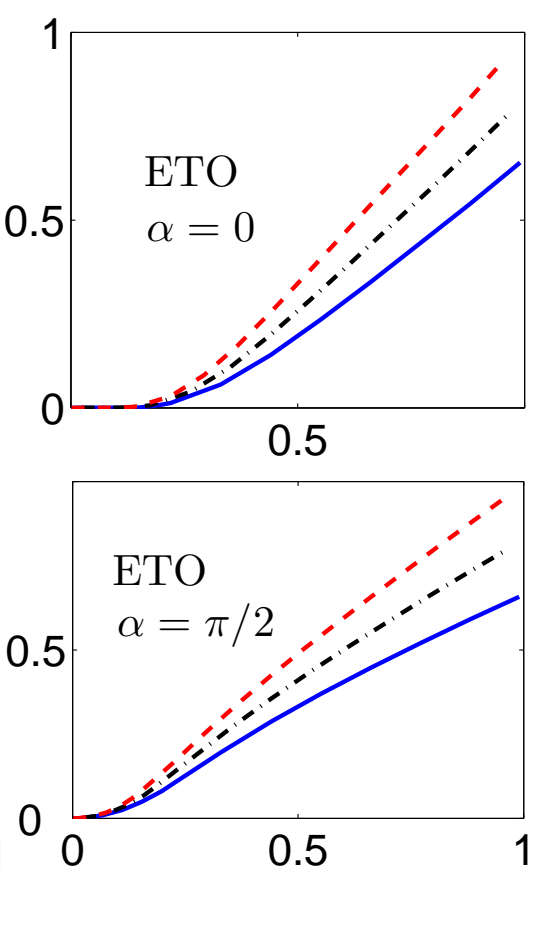

FIG. 3: (Color online) Plot of $\kappa$ for isotropic, even parity superconductors and odd parity superconductors for both even and odd frequency pairing. A power-law dependence with exponent $\simeq 1$ is observed for both of the odd frequency symmetries (see insets).

Consider first the left column of Fig. 2 where we have plotted $G$ as a function of bias voltage for the even-parity symmetries. In the even-frequency case, the usual singularity at $e V=A_{0} \Delta_{0}$ is present. In the odd-frequency case, we see a qualitatively different behaviour of the conductance. First of all, $G$ at low bias voltage is greatly enhanced compared to the even-frequency case for $A_{0}<1$, and the formation of a zerobias conductance peak is clearly seen at $A_{0}=1$. For $A_{0}>1$, the conductance is similar to the even-frequency case for a reduced value of the gap. This may be understood as follows. For $A_{0}<1$, the inequality $\varepsilon>\Delta(\varepsilon)$ is satisfied for all $\varepsilon$ with our choice of gaps (Tab. I). This corresponds to gapless superconductivity. For $A_{0}>1$, the gap becomes larger than $\varepsilon$ below a certain (finite) value of $\varepsilon$, similar to the BCS-gap.

The middle and right columns of Fig. 2] shows $G$ as a function of bias voltage for the odd-parity symmetries. In the OSO 
case, a gap like structure is seen at $\alpha=0$. This is because ESE pairing is induced near the interface due to the sign change of the pair potential. 5 This ESE pairing is responsible for the gap like structure of the conductance spectra, similar to the ESE case in Fig. 2. In contrast, OSO pairing remains near the interface at $\alpha=\pi / 2$. Thus, a zero-bias conductance peak is seen. On the other hand, in the ETO case at $\alpha=0$, a zerobias conductance peak appears due to the induced OTE pairing near the interface ${ }^{\frac{5}{}}$, similar to the OTE case in Fig. 2. At $\alpha=\pi / 2$, ETO pairing survives near the interface and hence the even frequency character of the pair amplitude results in a V-like shape of the spectra. Interestingly, OSO and ETO cases have the opposite tendency although their $\vartheta$-dependencies are the same. Furthermore, the sign change of the gap produces a qualitative difference in the spectra between OTE and OSO with $\alpha=0$ junctions. Thus, $G$ is phase sensitive not only in even frequency superconductor junctions ${ }^{17}$, but also in odd frequency superconductor junctions.

We next investigate the thermal conductance $\kappa$, shown in Fig. 3. The left column corresponds to the even parity case, where the usual exponential dependence on $T$ is recovered for the ESE case ${ }^{34}$. In the OTE case, $\kappa$ mimics the ESE case for $A_{0}>1$ just as for the charge conductance. Otherwise, power-law dependence with exponent $\simeq 1$ is observed due to the node of the gap at zero energy. Thus, the nodes in the frequency domain of an isotropic odd-frequency superconductor causes $\kappa$ to behave like in an anisotropic even-frequency superconductor. In the middle and right columns of Fig. 3, we give $\kappa$ in the odd parity case. The well-known result of exponential dependence for $\alpha=0$ is recovered in the ETO case. The OSO case again displays power-law behaviour similar to the OTE case for $A_{0}<1$. However, the exponential dependence again occurs for $A_{0}>1$ in the OSO case with $\alpha=0$. When $\alpha=\pi / 2$, there is exclusively power-law dependence, with exponent $\simeq 1$. While the OTE case only has nodes in energy, the OSO case has both nodes in energy and in $\mathbf{k}$-space, but this does not appear to influence the exponent of the power-law dependence.

\section{SUMMARY}

In summary, we have studied quantum transport in a normal metal/superconductor junction, considering how a bulk odd frequency symmetry in the superconductor is manifested in the electrical and thermal conductance of the junction. The odd frequency symmetry is found to display qualitatively distinct behaviour from the even frequency case. This reflects the fact that the electrical conductance is sensitive to the presence of odd frequency pairing at the interface, whereas the low temperature behavior of the thermal conductance reflects the node of the gap in the frequency domain. Moreover, one may distinguish the even and odd parity cases for an odd frequency symmetry (OTE and OSO, respectively) by means of their different characteristic tunneling spectra. Our predictions should be useful for a wide range of experimental techniques, and are thus a helpful tool in identifying the possible existence of bulk odd frequency superconductors, with $\mathrm{CeCu}_{2} \mathrm{Si}_{2}$, and $\mathrm{CeRhIn}{ }_{5}$ currently presenting themselves as the most promising candidates.

\section{Acknowledgments}

J.L. and A.S. were supported by the Research Council of Norway, Grants No. 158518/431 and No. 158547/431 (NANOMAT), and Grant No. 167498/V30 (STORFORSK). T.Y. acknowledges support by JSPS. T.Y and Y.T were supported by Grant-in-Aid for Scientific Research (Grant Nos. 17071007) from the Ministry of Education, Culture, Sports, Science and Technology of Japan. The authors acknowledge A. Balatsky for helpful comments. Y.T. would like to thank K. Miyake, H. Kohno and Y. Fuseya for their valuable dicussions. J.L. acknowledges K. Yada for clarifying comments on the odd-frequency pairing potential.
${ }^{1}$ F. S. Bergeret, A. F. Volkov, and K. B. Efetov, Phys. Rev. Lett. 86, 4096 (2001); A. F. Volkov, F. S. Bergeret, and K. B. Efetov, Phys. Rev. Lett. 90, 117006 (2003).

2 V. L. Berezinskii, JETP Lett. 20, 287 (1974).

3 R.S. Keizer, S. T. B. Goennenwein, T. M. Klapwijk, G. Miao, G. Xiao, A. Gupta, Nature 439, 825 (2006); I. Sosnin, H. Cho, V. T. Petrashov, and A. F. Volkov, Phys. Rev. Lett. 96, 157002 (2006).

${ }^{4}$ V. Braude and Yu. V. Nazarov, Phys. Rev. Lett. 98, 077003 (2007); Y. Asano, Y. Tanaka and A.A. Golubov, Phys. Rev. Lett. 98, 107002 (2007).

5 Y. Tanaka, A. A. Golubov, S. Kashiwaya, and M. Ueda, Phys. Rev. Lett. 99, 037005 (2007).

${ }^{6}$ M. Eschrig, T. Lofwander, Th. Champel, J.C. Cuevas, and G. Schon, J. Low Temp. Phys. 147457 (2007).

7 Y. Tanaka, Y. Tanuma and A. A. Golubov, Phys. Rev. B 76, 054522 (2007).

8 Y. Asano, Y. Tanaka, A. A. Golubov, S. Kashiwaya, Phys. Rev. Lett. 99, 067005 (2007)
9 Y. Tanaka, Y.V. Nazarov, A.A. Golubov, and S. Kashiwaya, Phys. Rev. B 69, 144519 (2004); Y. Tanaka and S. Kashiwaya, Phys. Rev. B 70, 012507 (2004); Y. Tanaka, S. Kashiwaya and T. Yokoyama, Phys. Rev. B 71,094513 (2005).

${ }^{10}$ Y. Tanaka, Yu. V. Nazarov, and S. Kashiwaya, Phys. Rev. Lett. 90, 167003 (2003).

11 A. Balatsky and E. Abrahams, Phys. Rev. B 45, 13125 (1992); E. Abrahams, A. Balatsky, D. J. Scalapino and J. R. Schrieffer, Phys. Rev. B 52, 1271 (1995).

12 P. Coleman, E. Miranda and A. Tsvelik, Phys. Rev. B 49, 8955 (1994); P. Coleman, A. Georges and A. M. Tsvelik, J. Cond. Matter 9345 (1997).

13 Y. Fuseya, H. Kohno and K. Miyake, J. Phys. Soc. Jpn. 72, 2914 (2003); G. Q. Zheng, et al., Phys. Rev. B, 70, 014511 (2004); S. Kawasaki et al., Phys. Rev. Lett. 91, 137001 (2003).

14 Y. Tanaka and A. A. Golubov, Phys. Rev. Lett. 98, 037003 (2007); Ya. V. Fominov, JETP Letters 86, 732 (2007) [Pis'ma v ZhETF 86, 842 (2007)]. 
15 J. Linder, T. Yokoyama, and A. Sudbø, arXiv: 0712 .0134

${ }^{16}$ G. E. Blonder, M. Tinkham, and T. M. Klapwijk, Phys. Rev. B 25, 4515 (1982).

17 Y. Tanaka and S. Kashiwaya, Phys. Rev. Lett. 74, 3451 (1995); Y. Tanuma, Y. Tanaka and S. Kashiwaya, Phys. Rev. B 64, 214519 (2001).

18 Y. Kasahara, Y. Nakajima, K. Izawa, Y. Matsuda, K. Behnia, H. Shishido, R. Settai, and Y. Onuki, Phys. Rev. B 72, 214515 (2005).

${ }^{19}$ G. Seyfarth, J. P. Brison, M.-A. Masson, J. Flouquet, K. Izawa, Y. Matsuda, H. Sugawara, and H. Sato, Phys. Rev. Lett. 95, 107004 (2005).

${ }^{20}$ J. Y. Wei, N.-C. Yeh, D. F. Garrigus, and M. Strasik, Phys. Rev. Lett. 81, 2542 (1998).

${ }^{21}$ E. V. Bezuglyi and V. Vinokur, Phys. Rev. Lett. 91, 137002 (2003).

22 L. J. Buchholtz and G. Zwicknagl, Phys. Rev. B 23,5788 (1981); J. Hara and K. Nagai, Prog. Theor. Phys. 74, 1237 (1986); C.-R. Hu, Phys. Rev. Lett. 72, 1526 (1994).

${ }^{23}$ G. M. Eliashberg, Zh. Eksp. Teor. Fiz. 38, 966 (1960).
${ }^{24}$ J. W. Serene and D. Rainer, Phys. Rep. 101, 221 (1983).

25 N. Kopnin, Theory of Nonequilibrium Superconductivity, Oxford University Press, New York, (2001).

26 J. Rammer and H. Smith, Rev. Mod. Phys. 58, 323 (1986).

27 A. M. Zagoskin, Quantum Theory of Many-Body Systems, Springer, (1998).

${ }^{28}$ M. Vojta and E. Dagotto, Phys. Rev. B 59, R713 (1999).

29 J. E. Bunder and K. B. Efetov, Phys. Rev. B 70, 134522 (2004).

${ }^{30}$ Y. Tanaka, A. A. Golubov, and S. Kashiwaya, Phys. Rev. B 68, 054513 (2003).

31 T. Yokoyama, Y. Tanaka, A. A. Golubov, and Y. Asano, Phys. Rev. B 72, 214513 (2005).

32 T. Yokoyama, Y. Tanaka, A. A. Golubov, J. Inoue, and Y. Asano, Phys. Rev. B 71, 094506 (2005).

${ }^{33}$ Ya. V. Fominov, A. F. Volkov, and K. B. Efetov, Phys. Rev B 75, 104509 (2007).

34 A. F. Andreev, Sov. Phys. JETP 19, 1228 (1964). 